\title{
Impacto de la donación renal de vivo en la evolución de la función renal del donante
}

\author{
Prieto-Matienzo Laura 1, *; Gómez-Dos-Santos Victoria ${ }^{2}$; Burgos-Revilla Francisco Javier ${ }^{3}$ \\ 1 Máster en Medicina, Universidad de Alcalá \\ 2 Servicio de Urología, Hospital Universitario Ramón y Cajal, IRYCIS, Universidad de Alcalá; \\ https://orcid.org/0000-0003-2968-148X \\ 3 Servicio de Urología, Hospital Universitario Ramón y Cajal, IRYCIS, Universidad de Alcalá \\ * Autor correspondencia: lauraprieto46@gmail.com.org;
}

DOI: https://doi.org/10.37536/RIECS.2020.5.1.187

Recibido: 07/02/2020; Aceptado: 20/04/2020; Publicado: 30/05/2020

Resumen: Introducción: El trasplante renal de donante vivo representa la mejor opción terapéutica para los pacientes con Insuficiencia Renal Crónica Terminal (IRC). Sin embargo, la nefrectomía conlleva ciertos riesgos a corto y largo plazo para el donante vivo. En este estudio queremos evaluar el impacto de la donación sobre la función renal del donante. Material y Métodos: Evaluamos retrospectivamente a los donantes vivos renales en el periodo comprendido entre 2010 y 2018 en el Hospital Universitario Ramón y Cajal. Se realizó un análisis descriptivo de la muestra y se usó el análisis de varianza (ANOVA) para comparar la edad y el IMC con el aclaramiento medido en los distintos momentos del tiempo. Así mismo, se realizó la curva de supervivencia de Kaplan-Meier en función del sexo para ver cuántos pacientes desarrollaban IRC post-nefrectomía. Resultados: De los 39 pacientes, al año de la nefrectomía el 34,4\% presentaron insuficiencia renal definida como un aclaramiento $<60 \mathrm{ml} / \mathrm{min} / 1,73 \mathrm{~m} 2$. Ningún paciente desarrolló IRCT. Los pacientes con mayor edad tuvieron un mayor deterioro de la función renal. No se demostró mayor deterioro de la función renal atendiendo al sexo y en los sujetos con IMC más alto. Conclusiones: La edad parece ser un factor de riesgo para la IRC mientras que el IMC y el sexo en la población estudiada, no parece que provoquen un mayor riesgo en el deterioro de la tasa de filtrado glomerular.

Palabras Clave: Insuficiencia renal crónica terminal, Trasplante renal, Donante renal vivo, Enfermedad renal crónica, Función renal.

\begin{abstract}
Background: Living donor kidney transplantation represents the best therapeutic option for patients with chronic terminal renal failure. However, nephrectomy bears certain short- as well as long-term risks for the living donor. In this study we want to evaluate the impact of donation on donor renal function. Materials and Methods: We retrospectively evaluated live kidney donors in the period between 2010 and 2018 at the Ramón y Cajal University Hospital. A descriptive analysis of the sample was carried out and it was used in analysis of variance (ANOVA) to compare the age and the BMI with the clearance measured at the different moments of time. Likewise, the KaplanMeier survival curve was performed according to sex to see how many patients developed postnephrectomy renal failure (CKD). Results: Of the 39 patients, one year after the nephrectomy, $34.4 \%$ had renal failure $(<60 \mathrm{ml} / \mathrm{min} / 1.73 \mathrm{~m} 2)$. No patient ended up on ESKD. The older patients had a greater deterioration of renal function. There is no greater deterioration of renal function in subjects with higher BMI. Conclusions: Age seems to be a risk factor for renal failure while BMI and sex do not seem to cause a greater risk in the deterioration of the glomerular filtration rate.
\end{abstract}

Key words: End stage renal disease, Kidney transplantation, Living donor kidney transplantation, Chronic kidney disease, Kidney function. 


\section{Introducción}

La Insuficiencia Renal Crónica Terminal es considerada el estadio más grave de la Enfermedad Renal Crónica. Según el Registro de la Sociedad Española de Nefrología de 2016, la prevalencia global de IRC en nuestro país fue de 1.233,5 pacientes por millón de población, de los cuales el 52,4\% tienen un trasplante renal funcional, el $42,2 \%$ está en hemodiálisis y el 5,5\% en diálisis peritoneal; siendo sin duda la mejor opción terapéutica el trasplante renal (TR) [1].

A lo largo de la historia en nuestro país, el TR ha ido evolucionando desde que en 1989 se crease la Organización Nacional de Trasplantes (ONT). Inicialmente la actividad de trasplante fue una actividad dependiente del donante fallecido y la comunidad trasplantadora era reticente a someter a una persona sana a un procedimiento quirúrgico. A partir del año 2000, se pone de manifiesto la necesidad de implementar nuevas estrategias, que incluyen, los donantes con criterios expandidos y los donantes vivos, debido a la escasez de órganos y falta de donantes cadáver [2-5].

El trasplante de donante vivo ha ido creciendo progresivamente conforme han ido pasando los años, alcanzando en el año 2014 la cifra más alta de nuestra historia, con 423 trasplantes renales de donante vivo (que supuso un 18\% de la actividad de trasplante renal) [6-7]. Sin embargo, en los últimos años ha presentado un descenso, debido a un nuevo incremento en el número de donantes fallecidos, fundamentalmente a expensas de los donantes en asistolia [6-7].

Así mismo, en Reino Unido el número de donantes fallecidos por asistolia y muerte cerebral ha ido aumentando año tras año, siendo en 2017-2018 su cifra más alta con 619 y 955 donantes, respectivamente. Además, el número de donantes vivos ha caído en los últimos 5 años, de un máximo de 1.148 donantes en 2013-2014 a 1.051 en 2017-2018. A pesar de ello, en comparación con el año pasado, en Reino Unido hubo un aumento del 1\% en donantes vivos en 2017-2018 [8].

Por otro lado, el donante vivo ha aumentado en Europa progresivamente entre 2004 y 2013, incrementándose en un 86\%. En 2013 se realizaron en la Unión Europea 24.082 trasplantes renales, de los cuales el 30\% fueron de donante vivo. [9] Además, entre 2009 y 2015 se llevó a cabo el Plan de Acción Europeo sobre Donación y Trasplante de Órganos que provocó, a su vez, un aumento en el número de donantes de órganos, pasando de 12.369 en 2008 a 14.953 en 2015. [10] A pesar de ello, aún existen diferencias sustanciales, entre los países de la Unión Europea, en la proporción de trasplante de donantes vivos frente a donantes fallecidos. Un ejemplo de ello se muestra en las estadísticas recogidas por el Registro ERA-EDTA de 2016, en el que el 100\% de los trasplantes realizados en Georgia, Islandia, Macedonia y Montenegro fueron de donante vivo, mientras que en países como Eslovenia o Bielorrusia este porcentaje estuvo tan sólo en un 4\% [11].

Dentro de la donación de vivo existen dos tipos principales:

- Donación directa: existe un vínculo afectivo entre donante y receptor.

- Donación indirecta: no existe vínculo entre donante y receptor [12].

La donación de vivo presenta numerosas ventajas frente al donante fallecido. Proporciona un paciente con un excelente estado de salud, con menos patología asociada y con un injerto de mejor calidad, así como una menor duración de los tiempos de isquemia y del tiempo de diálisis [2,12]. Según los datos de la Organ Procurement Transplant Network la supervivencia del injerto renal al año es del $89 \%$ para los trasplantes renales de cadáver frente al $95,1 \%$ para los realizados con donante vivo, siendo estas diferencias más significativas a los cinco años, con supervivencias del $66,5 \%$ frente al 79,7\% respectivamente [13]. Igualmente, un estudio descriptivo retrospectivo de la supervivencia de los injertos, realizado en el Hospital Universitario Puerta del Mar en Cádiz, entre 2005 y 2014, presentó resultados similares siendo la supervivencia del injerto de 90,1\% para donante cadáver y de 93,6\% para donante vivo [14]. Otro estudio observacional del registro UNOS, evidenció que la supervivencia del injerto de donante vivo al año era mejor que la del donante cadáver $(94,8 \%$ frente al 90,6\% respectivamente) [15]. Por otra parte, varios estudios de cohorte retrospectivos evidenciaron que el trasplante preventivo se asocia con una supervivencia superior del injerto en comparación con la diálisis previa al trasplante, posibilidad que otorga el donante vivo [15-16]. Del mismo modo, existe una clara asociación entre el tiempo prolongado de isquemia y los resultados adversos del injerto en 
el trasplante renal de donante fallecido. Cuanto mayor es el tiempo de isquemia, en particular $>4-8$ horas, mayor es el riesgo de peor función del injerto y a la larga mayor pérdida de este [17-18].

Así mismo, el donante vivo permite programar el trasplante e iniciar la inmunosupresión del receptor de forma anticipada, disminuyéndose el riesgo de rechazo. Todo lo anterior conlleva una mejor supervivencia del injerto y del paciente a largo plazo.

En los últimos años se han hecho varios estudios que han llegado a la conclusión de que, en comparación con las poblaciones sanas no donantes, los donantes vivos de riñón no tienen mayor riesgo de presentar más enfermedades crónicas importantes, con la excepción de ERC, aunque se vio que el riesgo absoluto de esta enfermedad sigue siendo bajo. Así mismo se han atribuido tasas superiores de supervivencia del paciente y del injerto ajustada a 5 años mayores para los trasplantes de donantes vivos en comparación con los trasplantes de donantes fallecidos: 94.6\% (IC 95\% 94.195.1) versus $91.9 \%$ (IC 95\% 91.6-92.3) en relación a la supervivencia del paciente y 86.7\% (IC 95\% 85.987.4) versus $80.9 \%$ (IC 95\% 80.4-81.4) para la supervivencia del injerto al año en 2016, siendo los datos bastante similares en 2017 [19-23]. Además, según los datos recogidos por la ONT en su registro de donantes vivos de 2010-2015 la supervivencia de los donantes vivos al año fue del 99,995\% y del 99,8\% a los 4 años. La mediana de seguimiento del total de donantes fue de 24 meses, observándose una mediana de TFG de $60 \mathrm{ml} / \mathrm{min}$ medida mediante CKDEPI [24]. De igual forma, los datos aportados por la OPTN/SRTR de 2017 también evidenciaron una mayor tasa de supervivencia del injerto ajustada a 10 años mayor para los trasplantes de donante vivo frente a los trasplantes de donantes fallecidos: $65,9 \%$ versus $50,3 \%$ [25]. Las principales razones que explican este hecho son que el donante vivo es exhaustivamente estudiado y seleccionado entre individuos sanos y sus órganos no están expuestos a la inestabilidad hemodinámica, la sepsis, ni los agentes nefrotóxicos como lo están los de los donantes fallecidos en muerte encefálica. Además, no sufren los efectos deletéreos de la muerte encefálica y tienen cortos períodos isquemia fría antes del implante.

Por el contrario, la nefrectomía supone un riesgo de desarrollar ERC, bien a través de la propia nefrectomía o del desarrollo de complicaciones que son factores de riesgo para su aparición (HTA, microalbuminuria, obesidad). Algunos estudios como el de Ibrahim et al asociaron de manera significativa que una mayor presión sistólica y un IMC más alto conducían a una mayor pérdida de TFG, aumentando el riesgo de padecer ERC [26]. Otros estudios plantean la hipótesis de que los donantes con HTA en el momento de la donación pueden tener una reserva funcional renal reducida después de la donación, presumiblemente debido a una reducción de la capacidad vasodilatadora renal. Esto deteriora la respuesta compensatoria renal para adaptarse a un estado de riñón único y da como resultado una TFG más baja durante el seguimiento [27]. Por ello, se ha planteado una estrategia para determinar de forma óptima la probabilidad, posterior a la prueba, de que el TFG sea inferior a $60 \mathrm{ml} / \mathrm{min} / 1,73 \mathrm{~m} 2$. Consiste en la obtención del TFG con creatinina sérica y cistina $C$ antes de la visita al centro de donantes; de esta forma, los donantes con una muy alta probabilidad después de la prueba de entrar en ERC podrían ser excluidos en lugar de someterse a una evaluación completa [28].

Del mismo modo, otros estudios investigaron las causas más frecuentes de muerte en donantes vivos postrasplante entre 1987 y 2016 y plantearon algunas soluciones, que ha asumido la OPTN, para reducir el número de muertes prevenibles en donantes vivos. Aunque el riesgo de muerte por donación es de 1:3000-5000, entre las causas de muerte más frecuentes se encontraban la hemorragia y el suicidio. Entre las medidas que podían prevenir estas muertes se vio que tener un equipo de trasplantes formado en convertir una nefrectomía laparoscópica a abierta reducía el número de muertes, así como usar grapadora o suturar las ligaduras de vasos en vez de poner un clip. Respecto a los suicidios, se vio que el número era superior entre donantes no relacionados con el receptor. Se ha postulado que un estudio emocional más exhaustivo de los donantes y un seguimiento de los mismos más estrecho podría disminuir esta causa de muerte [29].

Por ello, aunque se necesitan más estudios, el donante vivo se podría considera el tratamiento de elección, dado que los resultados para receptores renales de donante vivo son muy prometedores $[5,12,24]$. 
Además, la donación conlleva un estudio exhaustivo del futuro donante, para determinar si es candidato o no a la nefrectomía. Dentro de él se engloba la necesidad de hacer estudios clínicos (anamnesis, exploración física), estudios analíticos (incluyen el tipaje HLA y las pruebas cruzadas) y estudios complementarios funcionales y anatómicos; que incluyen de forma específica la evaluación de la función renal del donante.

Para llevar a cabo la evaluación de la función renal, se utilizan métodos para medir el TFG, siendo el patrón de referencia la medición del aclaramiento mediante trazadores externos como la inulina, el iohexol o el iotalamato. Debido a que estas técnicas son largas y complejas, en la práctica clínica se utilizan fórmulas como la fórmula Modification of Diet in Renal Disease de 4 variables (MDRD4) y la fórmula Chronic Kidney Disease-Epidemiology Collaboration (CKD-EPI) para medir la TFG [30-31].

A pesar de lo anterior, la donación también presenta potenciales riesgos. El donante presenta un riesgo de mortalidad inmediata de aproximadamente $0,03 \%$, un riesgo de morbilidad que oscila entre un $0,6 \%$ y un $14 \%$ y según estudios recientes, un mayor riesgo de enfermedad renal después de la donación, incluyendo un pequeño aumento en el riesgo de IRC [32-33].

\section{Material y Métodos}

El estudio está constituido por el análisis retrospectivo de la cohorte prospectiva de donantes vivos renales, en el periodo comprendido entre 2010 y 2018 en el Hospital Universitario Ramón y Cajal (HURyC).

El estudio recibió la aprobación del Comité de Ética de Investigación Clínica (CEIC) del HURyC (249/18).

De forma adicional se llevó a cabo una revisión sistemática de la literatura, para lo cual se realizó una búsqueda bibliográfica entre 2009-2019, a través de la base de datos Pubmed y ScienceDirect, para encontrar los estudios más relevantes que documentaran la asociación entre donación renal de vivo y evolución de la función renal y desarrollo de IRC.

La selección de los donantes y sus correspondientes receptores se realizó por un nefrólogo y un urólogo en base a los criterios de donación de riñón establecidos en el protocolo de donante vivo del HURyC y sus datos clínicos, analíticos y radiológicos fueron revisados y recogidos en una hoja de cálculo de Microsoft Excel diseñada a tal efecto.

\subsection{Criterios selección donantes vivos}

Según el protocolo del donante vivo se excluyeron como donantes de riñón a aquellos que cumplían con los siguientes criterios: (1) Edad: $<18$ años; (2) Hipertensión que precise más de 1 fármaco para control o que tenga lesión de órganos diana; (3) Obesidad: IMC $>35$; (4) TFG $<80$ $\mathrm{ml} / \mathrm{min} / 1,73 \mathrm{~m} 2$ en donantes de menos de 60 años y $<60 \mathrm{ml} / \mathrm{min} / 1,73 \mathrm{~m} 2$ en donantes de edad superior a 60 años; (5)Proteinuria $>150 \mathrm{mg}$ en 24 horas ó microalbuminuria $>30 \mathrm{mg} / \mathrm{g}$ de creatinina; (6) Nefrocalcinosis o malformaciones urológicas mayores; (7) Enfermedad sistémica con posible afectación renal; (8) Cualquier enfermedad que requiera anticoagulación sistémica; (9) Abuso de drogas IV; (10) Embarazo; (11) Historia previa de neoplasia maligna o historia familiar de primer grado de cáncer renal; (12) Enfermedades infecciosas o contagiosas, como VIH, hepatitis o HTLV; (13) Psicosis mal controlada

\subsection{Seguimiento}

Todos los donantes se sometieron a una evaluación de la TFG, de creatinina y de proteinuria antes de la nefrectomía, y luego al mes, a los 6 meses y al año después de la nefrectomía. La TFG se calculó mediante la fórmula de MDRD4.

\subsection{Análisis Estadístico}

Se realizará un análisis descriptivo de la muestra mediante las frecuencias absolutas y relativas para las variables categóricas. Los datos continuos se presentan como media más desviación estándar. 
Para el análisis estadístico del TFG se utilizará el análisis de la varianza con un factor (ANOVA) tanto para comparar la edad como el IMC con el aclaramiento medido en los distintos momentos del tiempo especificados anteriormente. Los pacientes fueron categorizados según su IMC (se clasificó según el Sistema de la OMS en normopeso-Grupo $1(\geq 18.50-24.99 \mathrm{~kg} / \mathrm{m} 2)$, sobrepeso-Grupo 2 ( $\geq 25$ $29.99 \mathrm{~kg} / \mathrm{m} 2)$ y obesidad-Grupo $3(\geq 30 \mathrm{~kg} / \mathrm{m} 2)$ ) y según la edad (Grupo $1(>30-\leq 45)$ años, Grupo 2 (>45- $\leq 55)$, Grupo $3(>55))$.

También se categorizaron según el grado de IRC basándonos en el sistema KDIGO en 4 grupos (Grupo 1: categoría G3b-FG entre 30- $\leq 44 \mathrm{ml} / \mathrm{min} / 1,73 \mathrm{~m} 2$; Grupo 2: categoría G3a-FG entre $44-\leq 59$ $\mathrm{ml} / \mathrm{min} / 1,73 \mathrm{~m} 2$; Grupo 3: categoría G2-FG entre $59-\leq 89 \mathrm{ml} / \mathrm{min} / 1,73 \mathrm{~m} 2$ y Grupo 4: categoría G1-FG $>89 \mathrm{ml} / \mathrm{min} / 1,73 \mathrm{~m} 2)$.

Así mismo, la comparación del desarrollo de IRC entre hombres y mujeres se realizó con el test U de Mann-Witney. La supervivencia libre de IRC, definida como FG $\leq 60 \mathrm{ml} / \mathrm{min} / 1.73 \mathrm{~m} 2$, se analizó mediante la curva de Kaplan-Meier en función del sexo. Se tomará como IRC. Se consideró estadísticamente significativa $\mathrm{p}<0.05$.

También realizamos un análisis de T-student para datos pareados tanto en el grupo de HTA, como en el grupo de dislipemia.

Para este estudio todos los análisis estadísticos se realizaron con el paquete de software de Windows SPSS versión 23.0.

\section{Resultados}

\subsection{Características de donantes y receptores}

Se realizaron 39 trasplantes renales de donante vivo en el HURyC entre 2010 y 2018. La edad media de los donantes fue de 52,7 $\pm 10,2$ años con una proporción de hombres y mujeres de 38,5\% (15/39) frente al 61,5\% (24/39) respectivamente (Figura 1).

También se analizó la lateralidad, siendo el riñón izquierdo el más frecuentemente obtenido con un 97,4\% (38/39) frente al 2,6\% (1/39) del lado derecho. Así mismo, se analizó el número de donantes que hubo de cada Comunidad Autónoma resultando el 15,4\% (6/39) de los donantes procedentes de otras Comunidades Autónomas, en el contexto del programa CSUR de Centros, Servicios y Unidades de Referencia del Ministerio de Sanidad, siendo el 10,3\% (4/39) de Castilla La Mancha, el 2,6\% (1/39) de Andalucía y el 2,6\% (1/39) de Castilla y León. Además, se examinó la relación entre el donante y el receptor y se obtuvo que el 35,9\% (14/39) fueron madres que donaron a sus hijos, el 25,6\% (10/39) fueron entre hermanos/as, el 20,5\% (8/39) fueron entre parejas, el 10,3\% (4/39) fueron padres que donaron a sus hijos, el 5,1\% (2/39) fueron donantes cruzados y finalmente, en el 2,6\% (1/39) no existía parentesco entre donante y receptor (donante altruista). De igual forma, se investigó sobre los grupos sanguíneos de los donantes, siendo el 51,3\% (20/39) grupo 0, el 41,0\% (16/39) grupo A y sólo el 7,7\% (3/39) grupo B. No hubo ningún donante del grupo AB (Figura 1).

Por otro lado, un 56,4\% de los donantes presentaron alguna comorbilidad previa a la nefrectomía, siendo las más importantes en el contexto que nos ocupa, la HTA en un 12,6\% (5/39), la dislipemia en un 10,3\% (4/39) y la intolerancia hidrocarbonada en un 2,6\% (1/39). 

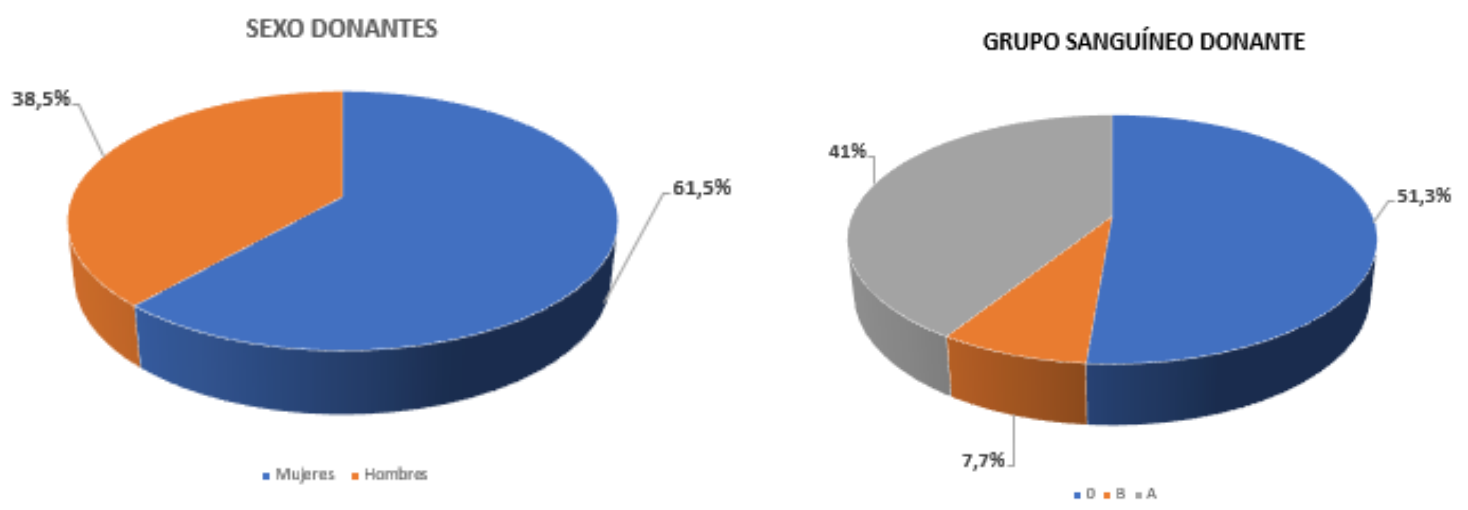

Figura 1 Trasplante renal vivo. Sexo y Grupo sanguíneo de donantes

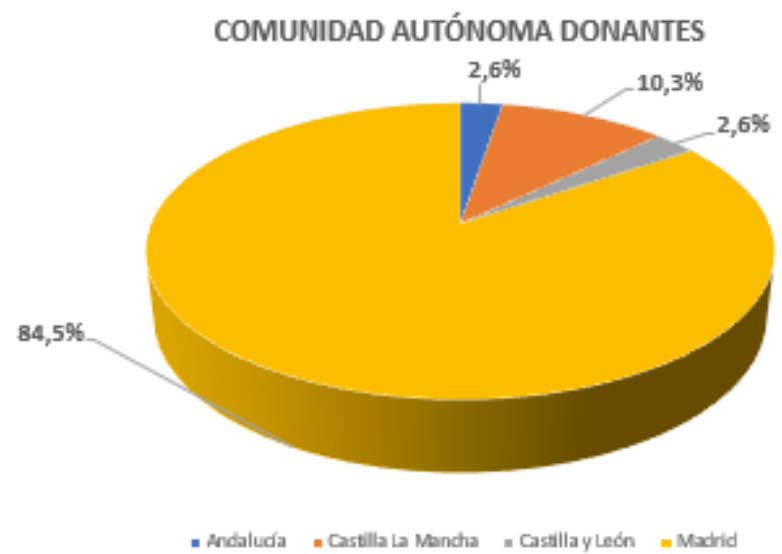

Figura 2 Distribución en porcentajes de la procedencia de los donantes y los receptores.

\subsection{Aclaramiento renal en el tiempo según la edad y el IMC}

Respecto a los donantes, para poder realizar el ANOVA se crearon varios grupos de edad e IMC para valorar si existían diferencias significativas respecto al aclaramiento renal tras la nefrectomía.

Respecto a la edad de los donantes, el 23,1\% (9/39) tenían edades comprendidas entre los 30 y los 45 años (Grupo 1); el 33,3\% (13/39) tenían edades comprendidas entre los 45 y los 55 años (Grupo 2) y el $43,6 \%$ (17/39) era mayor de 55 años (Grupo 3).

En cuanto al IMC el 53,8\% (21/39) de los donantes presentaban normopeso; el 30,8\% (12/39) tenían sobrepeso y el 15,6\% (6/39) tenían obesidad, aunque siempre con un IMC $<35$ puesto que es criterio de exclusión.

Se realizó un ANOVA de medidas repetidas de una vía para comparar por rangos de edad el aclaramiento antes de la nefrectomía, al mes, a los 6 meses y al año después de la misma. Se comprobó que existen diferencias significativas entre los grupos de edad de 30-45 años frente a los de $>55$ años tanto en el aclaramiento renal inicial, como el medido al mes, a los 6 meses y al año después de la nefrectomía. Sin embargo, entre los grupos de 30-45 frente a 45-55 y los de 45-55 frente a >55 no hubo diferencias significativas. (Figura 3) 


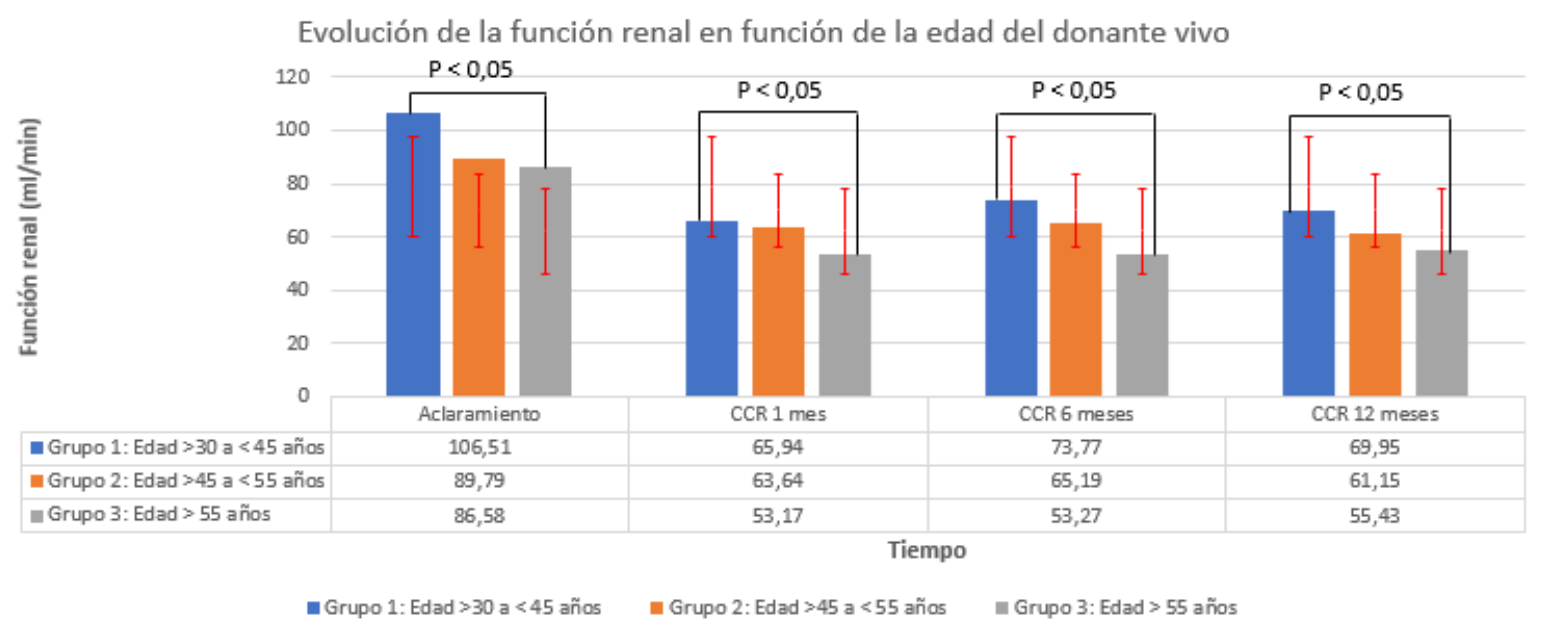

Figura 3 Evolución de la función renal en función de la edad del donante vivo

Del mismo modo, se realizó un ANOVA de medidas repetidas de una vía para comparar por rangos de IMC el aclaramiento antes de la nefrectomía, al mes, a los 6 meses y al año después de la misma, no existiendo diferencias significativas entre los grupos (Figura 4)

Evolución de la función renal en el tiempo en función del IMC del donante vivo

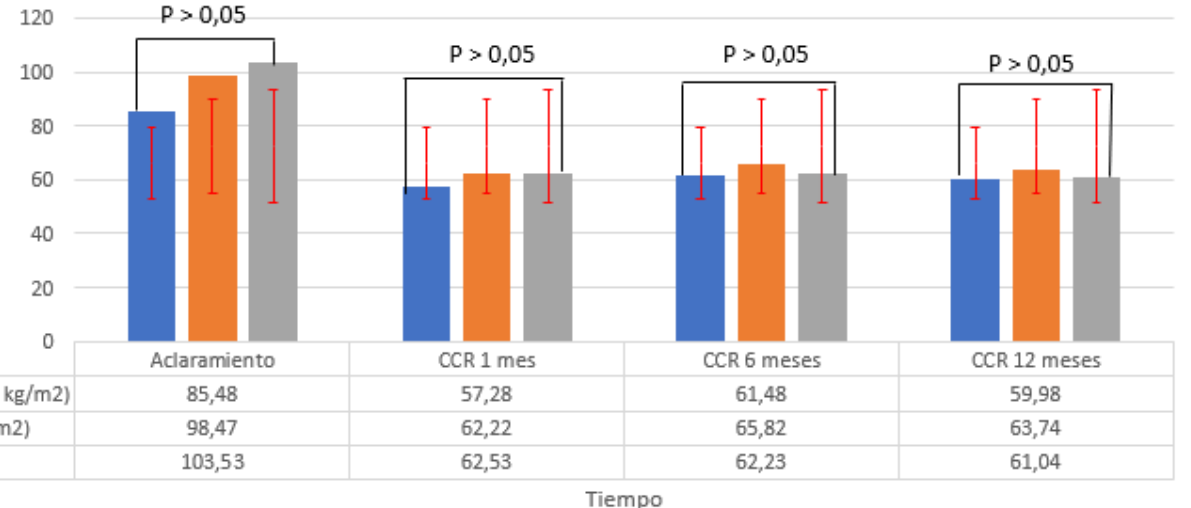

- Normopeso-Grupo $1(\geq 18.50-24.99 \mathrm{~kg} / \mathrm{m} 2)$

= Sobrepeso-Grupo $2(\geq 25-29.99 \mathrm{~kg} / \mathrm{m} 2)$

n Obesidad-Grupo $3(\geq 30 \mathrm{~kg} / \mathrm{m} 2)$

Figura 4 Evolución de la función renal en el tiempo en función del IMC del donante vivo

\subsection{Evolución función renal por sexos}

También se comparó cuántos pacientes quedaron en IRC $(<60 \mathrm{ml} / \mathrm{min} / 1,73 \mathrm{~m} 2)$ al mes de la nefrectomía, suponiendo estos un 48,7\% (19 casos de 39). Al año de la nefrectomía, tan sólo el 34,4\% de los casos (11/32) tuvieron IRC, aunque se perdió el aclaramiento renal al año de 7 casos $(17,9 \%$ de la muestra). De los 11 pacientes que presentaron IRC al año, el 45\% fueron hombres y el 55\% mujeres. No se apreciaron diferencias significativas entre hombres y mujeres (Figura 5). A pesar de los datos anteriores, ningún paciente desarrolló IRCT. 


\section{Curva Supervivencia}

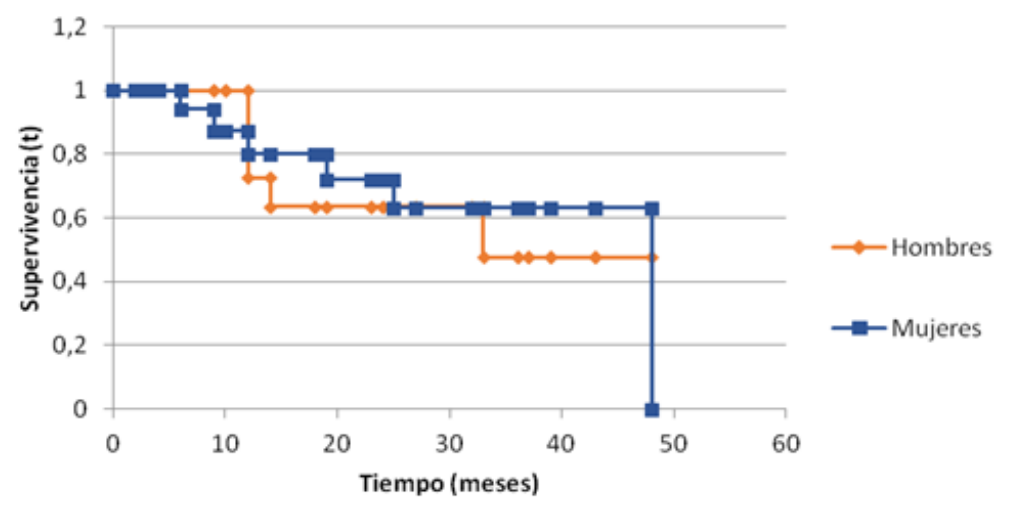

\begin{tabular}{|c|c|c|c|c|c|c|}
\hline Tiempo (meses) & 0 & 10 & 20 & 30 & 40 & 50 \\
\hline Hombres (n) & 14 & 12 & 7 & 5 & 1 & 0 \\
\hline Mujeres (n) & 18 & 11 & 8 & 4 & 1 & 0 \\
\hline
\end{tabular}

Figura 5 Evolución de la función renal postnefrectomía en hombres y mujeres a lo largo del tiempo

Por otro lado, la media del aclaramiento basal de los hombres fue de 100,3 $\pm 26 \mathrm{ml} / \mathrm{min} / 1,73 \mathrm{~m} 2$ mientras que en las mujeres fue de $87,2 \pm 13,2 \mathrm{ml} / \mathrm{min} / 1,73 \mathrm{~m} 2$. En el primer mes tras la nefrectomía ambas medias descendieron, aunque sólo las mujeres estuvieron por debajo de $60 \mathrm{ml} / \mathrm{min} / 1,73 \mathrm{~m} 2$. A los 6 meses y al año el TFG mejoró para ambos grupos, siendo la mejoría más importante en las mujeres (Figura 6).

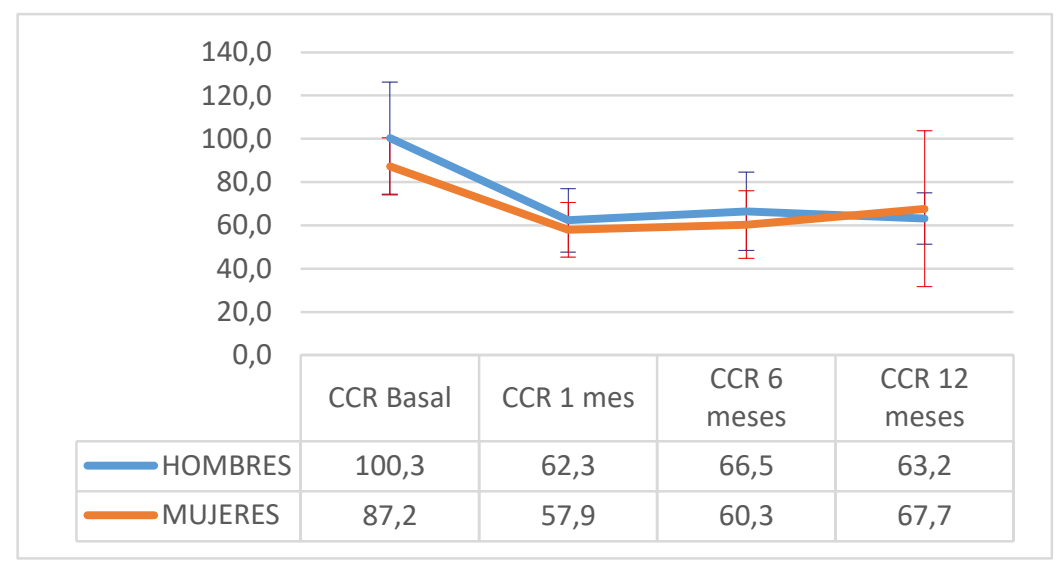

Figura 6 Evolución del TFG de los donantes, según el sexo, a lo largo del tiempo

También se evaluó cuántos pacientes presentaban HTA o dislipemia y si existía mayor predisposición a IRC, en los pacientes que presentaban alguna de las anteriores patologías. En nuestra muestra, el 10,3\% (4/39) de los pacientes presentaron dislipemia, el 12,8\% (5/39) presentaron HTA y un 2,6\% presentó ambas patologías de forma previa a la nefrectomía.

En el análisis univariante, los pacientes con HTA $(95,6 \pm 12,5 \mathrm{ml} / \mathrm{min} / 1,73 \mathrm{~m} 2$ vs $63,5 \pm 7,8$ $\mathrm{ml} / \mathrm{min} / 1,73 \mathrm{~m} 2)$ y/o dislipemia $(86,7 \pm 11,3 \mathrm{ml} / \mathrm{min} / 1,73 \mathrm{~m} 2$ vs $57,3 \pm 7,5 \mathrm{ml} / \mathrm{min} / 1,73 \mathrm{~m} 2)$ mostraron una disminución significativa del aclaramiento renal al año. 
Tabla I

\begin{tabular}{|c|c|c|}
\hline & $\begin{array}{c}\text { Aclaramiento al inicio } \\
(\mathbf{m l} / \mathbf{m i n} / \mathbf{1}, \mathbf{7 3 m} \mathbf{2})\end{array}$ & $\begin{array}{c}\text { Aclaramiento al año } \\
(\mathbf{m l} / \mathbf{m i n} / \mathbf{1}, \mathbf{7 3 m} \mathbf{m})\end{array}$ \\
\hline HTA & $95,6 \pm 12,5$ & $63,5 \pm 7,8$ \\
\hline Dislipemia & $86,7 \pm 11,3$ & $57,3 \pm 7,5$ \\
\hline
\end{tabular}

Por otra parte, 5/39 pacientes presentaron microhematuria definida como la presencia de $>3$ hematies por campo. En ningún caso se identificó patología urológica o nefrológica en los estudios complementarios pre-donación o en su evolución posterior.

Respecto a la microalbuminuria, el 17,9\% (7/39) presentaron determinaciones de microalbuminuria pre-donación entre $3-30 \mathrm{mg} / \mathrm{mmol}$; sin embargo, en ningún caso la microalbuminaruia se mantuvo en el periodo de seguimiento con determinaciones sistemáticamente $<3 \mathrm{mg} / \mathrm{mmol}$ al año de la cirugía. 6 pacientes presentaron cifras de microalbuminuria entre 3-30 $\mathrm{mg} / \mathrm{mmol}$ al año post-donación con una mediana de $8,5 \mathrm{mg} / \mathrm{mmol}$.

\section{Discusión}

El trasplante renal de donante vivo representó en España, en su momento de mayor auge, el $15,8 \%$ de los donantes, sin embargo, en los últimos años se ha asistido a un descenso progresivo en el número de donantes. En el año 2018, la Comunidad Autónoma con más trasplantes de donante vivo fue Cataluña con un 16,3 pmp seguida de Galicia con un 11.5 pmp, el País Vasco con un 5,9 pmp y Madrid con un 5,8 pmp. Dentro de la Comunidad de Madrid, el Hospital Universitario Doce de Octubre fue el hospital en el que más trasplantes de donante vivo se hicieron, representando el 57,9\% de los trasplantes de donante vivo de la Comunidad en 2018, seguido por el HURyC dónde se realizaron, el 13,2\% de los mismos en 2018 [38].

En este estudio, seguimos a 39 donantes renales de vivo después de su donación durante un año. Las características generales de los donantes de la serie estudiada fueron similares a las recogidas en los informes de la memoria de la ONT correspondiente a 2017 y en el informe del registro de donante vivo en España en 2018. Se observó que la mayoría de las personas que fueron donantes vivos eran adultos con una edad media de 52,0 años. Esto se corresponde con la edad media de los donantes incluidos en los informes de registro de donante vivo en España de 2018, siendo ésta de 53,8 años y de la memoria de 2017 de la ONT donde era de 52,9 años.

Además, en nuestro estudio casi el 62,0\% de los donantes eran mujeres, lo que se corresponde con los informes de registro de donante vivo en España de 2018, dónde el 68,0\% de los donantes vivos son mujeres y con la memoria de la ONT de 2017 donde el 64,0\% lo fueron. De igual forma, el grupo sanguíneo que fue más frecuente en nuestros donantes con un 49,6\% fue el grupo 0 , siendo este también el grupo más frecuente según los informes de registro de donante vivo en España de 2018 y de la memoria de la ONT de 2017. Sin embargo, en nuestro estudio la relación más frecuente entre donante y receptor fue entre madres que donaron a sus hijos seguidos de hermanos y parejas, mientras que en los informes de donantes vivos incluidos en RETReVi entre 2010 y 2015 la relación más frecuente entre donante y receptor fue esposo/a, seguida de madre/padre. Estos resultados son similares a los de la memoria de 2017 de la ONT y del registro de donante vivo de España de 2018 donde la relación entre donante y receptor más frecuente fue esposo/a seguido de madres y posteriormente entre hermanos. Estas diferencias podrían deberse a que nuestra población es pequeña, ya que sólo contamos con una muestra de 39 casos $[6,7,38]$.

Respecto a la TFG en función de la edad, los resultados del presente estudio revelaron que existen diferencias significativas. Los donantes mayores ( $>55$ años de edad) exhibieron un deterioro mayor de la función renal después de 1 año (86,58 vs 55,43 ml/min/1,73m2). Como en nuestro caso, en un estudio transversal de 573 donantes vivos de riñón mostraron que, en comparación con los donantes de 30 años, los donantes de 50 años presentaron una disminución más rápida de la función renal [39]. Otros estudios sugieren que la TFG disminuye después de los 35 años de forma lineal siendo más rápida en las mujeres que en los hombres [40]. Respecto a nuestros datos, es cierto que se 
puede apreciar un mayor descenso de la TFG en las mujeres, en el primer mes de la nefrectomía, pero no se pudo valorar en función de la edad y el sexo debido a la pequeña muestra de pacientes. Sin embargo, en otro estudio en el que se incluyeron 539 pacientes se evidenció que después de una disminución inicial de la función renal, no hubo una disminución acelerada de la misma ni en donantes jóvenes ( $<60$ años), ni en donantes mayores. Además, los donantes de mayor edad $(\geq 60)$ tuvieron una TFG más baja antes de la donación, pero no hubo diferencias en la disminución máxima media de TFG [41]. Apoyando lo anterior, Poggio y Rule realizaron un estudio de cohorte que sugiere que a medida que los sujetos envejecen, la TFG disminuye de manera acelerada independientemente de la nefrectomía, debido a un proceso fisiológico del envejecimiento normal [42].

Por otro lado, para la selección de nuestros donantes la TFG se calculó mediante la fórmula MDRD4. Sin embargo, la ecuación recomendada según los últimos estudios es la ecuación CKD-EPI y en los últimos años se han propuesto nuevas ecuaciones que incluyen marcadores endógenos como cistina C o la microglobulina beta 2. A pesar de lo anterior, la medición de la TFG con trazadores exógenos es el método de referencia para evaluar la función renal. El problema de utilizar estas ecuaciones en la selección de los donantes vivos es que estas herramientas de estimación y evaluación de la TFG se han realizado en pacientes con IRC por lo que no son muy válidas en donantes vivos de riñón [43]. Además, otro estudio determinó que las ecuaciones de estimación de las TFG basadas en creatinina, cómo es la ecuación de MDRD4, no eran fiables para la evaluación de los donantes obesos [44]. Finalmente, y respecto a las técnicas de evaluación de la función renal, la volumetría renal por tomografía computarizada (VRTC) ha demostrado proporcionar información tanto anatómica como funcional y, por tanto, es una técnica más precisa para la evaluación de la función renal individual y permite una predicción más fiable de la función renal postdonación [45-47].

En cuanto al IMC no existieron diferencias significativas en la determinación de TFG. Múltiples estudios [45-48] pusieron de manifiesto la ausencia de impacto de la obesidad en la función renal, entre ellos destaca el estudio retrospectivo de más de 5000 donantes renales vivos realizado por Reese et al [47]. Por el contrario, otros autores, observaron la asociación de un IMC alto ( $\geq 30 \mathrm{~kg} / \mathrm{m} 2)$ con una disminución más pronunciada de la función renal [35,38-39]. Locke et al. realizaron un estudio de cohorte de 119.769 donantes renales vivos en Estados Unidos donde se vio que, en comparación con los donantes vivos no obesos, los donantes obesos tenían un riesgo 1,9 veces mayor de ERC posterior a la donación y por cada aumento de una unidad en el IMC anterior a la donación por encima de $27 \mathrm{~kg} / \mathrm{m} 2$ hubo un aumento del 7,0\% en el riesgo de ERC post-donación [49]. Además, otros estudios demuestran que sería la obesidad visceral la que puede tener un impacto significativo en la función renal temprana después de la nefrectomía del donante [51].

Al año de la nefrectomía el $34,4 \%$ de los pacientes tuvo una TFG $<60 \mathrm{ml} / \mathrm{min} / 1,73 \mathrm{~m} 2$, lo que supuso una proporción alta de nuestra muestra. A pesar de ello, sólo un 6,2\% tuvo una TFG de $30-\leq$ $44 \mathrm{ml} / \mathrm{min} / 1,73 \mathrm{~m} 2$, no llegando ninguno a IRCT. La revisión de estos resultados debería hacer que considerásemos una modificación de las herramientas de evaluación de la función renal preoperatoria del donante, incluida la valoración anatómica, ya que la mayoría de los estudios que fueron analizados muestran que tan sólo un $8-21,0 \%$ de los pacientes quedan con una TFG $<60$ $\mathrm{ml} / \mathrm{min} / 1,73 \mathrm{~m} 2$ [52-53]. También debemos tener en cuenta que se perdió un 17,9\% de los datos del aclaramiento al año de la nefrectomía, lo que supone una limitación en nuestro estudio. Del mismo modo, el período de observación de la función renal de los donantes fue escaso (12 meses), por lo que sería una limitación añadida que no permitiría descartar una futura pérdida progresiva de la función renal.

Estudios similares de cohortes evaluaron también el papel de la HTA, la dislipemia y la hiperglucemia como factores de riesgo para desarrollar una IRC después de la nefrectomía en los donantes vivos. Ningún paciente desarrolló HTA de nueva aparición o empeoramiento de su control representado por un incremento en el requerimiento de tratamiento farmacológico, en el seguimiento post-nefrectomía, si bien como se ha hecho constar, el tiempo de seguimiento es limitado. Según los datos de la ONT 2010-2017, a los 6 años el 10,9\% de los pacientes registrados presentaron HTA de nueva aparición, controlándose el $50 \%$ con un solo fármaco. Al contrario que con la proteinuria, es destacable que la HTA de novo fue aumentando con el paso de los años [54]. 
Respecto a la presencia de microhematuria y proteinuria, el protocolo del hospital incluye como contraindicación relativa la "microhematuria persistente: No es contraindicación a la donación si tras estudios médicos y urológicos exhaustivos no se encuentra patología. No obstante, se ha objetivado en algunos estudios que el riesgo de insuficiencia renal se duplica en donantes con microhematuria persistente, lo que obliga a información clínica y a posible exclusión en donantes que son familiares directos de receptores con patología glomerular"

En relación con la microalbuminuria se consideró una contraindicación absoluta para la donación una microalbuminuria $>30 \mathrm{mg} / \mathrm{mmol}$ de acuerdo con las todas las guías clínicas. Cifras de microalbuminuria entre $3-30 \mathrm{mg} / \mathrm{mmol}$ tienen un significado controvertido, aunque se ha establecido que en la población general puede considerarse un factor de progresión de nefropatía e incrementar el riesgo cardiovascular. No existe un consenso general sobre la actuación requerida en el donante vivo renal si bien se recomienda su estudio específico.

\title{
5. Conclusiones
}

En conclusión, este estudio ha demostrado un deterioro a largo plazo de la TFG tras la donación en vivo en función de la edad. Los pacientes mayores ( $>55$ años) tienen un deterioro de la función renal mayor al año que los pacientes más jóvenes. Así mismo, ha demostrado que no existe mayor deterioro de la función renal en los sujetos con un IMC más alto, aunque los sujetos incluidos tenían un IMC $<35 \mathrm{~kg} / \mathrm{m} 2$ y que no hay diferencias estadísticamente significativas en función del sexo. Por otro lado, aunque la donación en vivo provoque una disminución en la TFG dejando a algunos pacientes en IRC, ninguno alcanza la situación de IRCT.

Agradecimientos: A los Dres. Sara Álvarez Rodríguez y Víctor Diez Nicolás por su facilitación al acceso de los datos clínicos.

Contribución de los autores: Diseño del estudio: Victoria Gómez Dos Santos y Francisco Javier Burgos Revilla. Recogida de datos: Laura Prieto Matienzo. Análisis de datos: Laura Prieto Matienzo y Victoria Gómez Dos Santos. Redacción y revisión del texto: Laura Prieto Matienzo, Victoria Gómez Dos Santos y Francisco Javier Burgos Revilla.

Conflictos de Intereses: Los autores no declaran conflicto de intereses.

\author{
Abreviaturas \\ Las siguientes abreviaturas son usadas en este manuscrito: \\ ANOVA: Análisis de varianza. \\ CKD-EPI: Chronic Kidney Disease-Epidemiology Collaboration. \\ IMC: Índice de Masa Corporal. \\ IRC: Insuficiencia Renal Crónica. \\ MAG3: gammagrafía con Tc-99m-mercaptoacetiltriglicina. \\ MDRD4: Modification of Diet in Renal Disease de 4 variables. \\ ONT: Organización Nacional de Trasplantes. \\ TEFG: Tasa Estimada Filtrado Glomerular. \\ TFG: Tasa de Filtración Glomerular medida. \\ TR: Trasplante Renal. \\ UNOS: United Network for Organ Sharing. \\ VRTC: Volumetría Renal por Tomografía Computarizada.
}




\section{Referencias Bibliográficas}

1. Informe de Diálisis y Trasplantes 2016. Sociedad Española de Nefrología (Internet). Disponible en: http://www.senefro.org/contents/webstructure/InformeREER_2016_BURGOS.pdf

2. Guiradi Perih L, Oppenheimer Salinas F. Trasplante renal de donante vivo. En: Lorenzo V, López Gómez JM (Eds) Nefrología al Día. http://dev.nefro.elsevier.es/es-monografias-nefrologia-dia-articulo-trasplanterenal-donante-vivo-144

3. Gozdowska J, Zatorski M, Torchalla P, Białek Ł, Bojanowska A, Tomaszek A, et al. Living-Donor Versus Deceased-Donor Kidney Transplantation: Comparison of Psychosocial Consequences for Recipients. Transplantation Proceedings 2016;48(5):1498-1505.

4. Aldabó Pallás T. Donante vivo. Legislación. Cuad Med Forense 2015;21(1-2):24-33

5. Domínguez-Gil B, de la Oliva V.M, Martín Escobar E, Cruzado J.M, Pascual J, Fernández Fresnedo G. Situación actual del trasplante renal de donante vivo en España y otros países: pasado, presente y futuro de una excelente opción terapéutica. Nefrología [Internet]. 2010 Dec 1 [cited 2019 Jan 2];30:3-13. Disponible en: http://www.revistanefrologia.com/es-situacion-actual-del-trasplante-renal-donante-vivo-espanaotros-paises-articulo-X0211699510051435

6. Dossier Trasplante Renal con Donante Vivo 2016. Ministerio de sanidad, servicios sociales e igualdad. [Internet]. Disponible en: htfile://C:/Users/laura/Downloads/DOSSIER\%20VIVO\%20RENAL\%20\%202016.pdf

7. Organización Nacional De Trasplantes [sitio en Internet]. Memoria Actividad de Donación y Trasplante Renal España 2017. [Actualizado 19 Julio 2018]. Disponible en:

http://www.ont.es/infesp/Memorias/Memoria\%20Renal\%202017_para\%20colgar.pdf

8. NHS Blood and Transplant [sitio en Internet]. Activity Report 2017-2018 Organ Donation and Transplant from the UK Transplant Registry. [Actualizado 8 Mayo 2018]. Disponible en:

https://nhsbtdbe.blob.core.windows.net/umbraco-assets/1848/transplant-activity-report-2017-2018.pdf

9. Comisión Europea [sitio en Internet]. Brussels: Journalist Workshop on Organ donation and transplantation. Recent Facts \& Figures [Citado 26 November 2014]. Disponible en: https://ec.europa.eu/health//sites/health/files/blood_tissues_organs/docs/ev_20141126_factsfigures_en.pdf

10. Bouwman R, Wiegers T, van Schoten S, Coppen R, Friele R. Study on the uptake and impact of the EU Action Plan on Organ Donation and Transplantation (2009-2015) in the EU Member States. European Comission. November 2017. Doi:10.2818/247136.

11. ERA-EDTA Registry. ERA-EDTA Registry Annual Report 2016. Amsterdam: Department of Medical Informatics, Amsterdam Unversity Medical Center, 2018. https://www.era-edtareg.org/files/annualreports/pdf/AnnRep2016.pdf

12. Srivastava T, Hariharan S, Alon U.S, McCarthy E.T, Sharma R, El-Meanawy A, et al. Hiperfiltrationmediated Injury in the Remaining Kidney of a Transplant Donor. Transplantation Journal 2018; 102(2):16241627.

13. Domínguez-Gil B, Pascual J. El trasplante renal de donante vivo en España: una gran oportunidad. Nefrología 2008;28(1):143-147.

14. Gil Barrera I, Velázquez Fernández A, Romero López R, Caro Torrejón M, Torres Arauz C, Moreno Rodríguez A. Trasplante renal donante cadáver versus donante vivo 2005-2014. Enferm Nefrol 2015; 18 Suppl (1): 74/79.

15. Luna E, Alonso Hernández A, Ramos Verde A, González Roncero F.M, Aguera M, Franco Esteve A et al. Marcadores de supervivencia pretrasplante del donante y del receptor. Nefrología Sup Ext 2015;6(2):37-67.

16. Haller MC, Kainz A, Baer H, Oberbauer R. Dialysis Vintage and Outcomes after Kidney Transplantation: A Retrospective Cohort Study. Clin J Am Soc Nephrol. 2017;12(1):122-130.

17. Salahudeen AK, Haider N, May W. Cold ischemia and the reduced long-term survival of cadaveric renal allografts. Kidney Int. 2004 Feb;65(2):713-8.

18. Krishnam A.R, Wong G, Chapman J.R, Coates P.T, Russ G.R, Pleass H, Russel C, et al. Prolonged Ischemic Time, Delayed Graft Function, and Graft and Patient Outcomes in Live Donor Kidney Transplant Recipients. American Journal of Transplantation. 2016 Sep;16(9):2714-23.

19. Hart A, Smith J.M, Skeans M. A, Gustafson S. K, Wilk A. R, Robinson A, et al. OPTN/SRTR 2016 Annual Data Report: Kidney. American Journal of Transplantation. 2018 Jan;18(Suppl 1):18-113. doi: 10.1111/ajt.14557 
20. O'Keeffe LM, Ramond A, Oliver-Williams C, et al. Mid- and Long-Term Health Risks in Living Kidney Donors: A Systematic Review and Meta-analysis. Ann Intern Med. 2018; (168):276-284. [Epub ahead of print 30 January 2018]. doi: https://doi.org/10.7326/M17-1235

21. Lanot A, Bouvier N, Chatelet V, Lecouf A, de Ligny BH, Tillou X. Devenir Des Donneurs Vivants De Rein Pour La Transplantation. Nephrologie et Therapeutique 2017 Nov;13(6):448-459. doi:10.1016/j.nephro.2017.02.011.

22. Organ donation and transplantation in Canada - system progress report2006-2015. Ottawa: Canadian Blood Services; 2016. Available: https://professionaleducation.blood.ca/en/organs-tissues (accessed 2017 Mar. 16).

23. ERA-EDTA Registry: ERA-EDTA Registry Annual Report 2017. Amsterdam UMC, location AMC, Department of Medical Informatics, Amsterdam, the Netherlands, 2019.

24. Registro Español de Trasplante Renal con Donante Vivo. Memoria de resultados 2010-2015. Disponible en: http://www.ont.es/infesp/Registros/INFORME\%20REGISTRO\%20DONANTE\%20VIVO\%2020102015_definitivo_Web.pdf

25. OPTN/SRTR 2017 Annual Data Report: Kidney. Available: https://srtr.transplant.hrsa.gov/annual_reports/2017/Kidney.aspx

26. Ibrahim H.N., Foley R.N., Reule S.A., Spong R., Kukla A., Issa N., Berglund D.M., Sieger G.K., Matas A.J. Renal Function Profile in White Kidney Donors: The First 4 Decades. J. Am. Soc. Nephrol. 2016;27:28852893. doi: 10.1681/ASN.2015091018.

27. Kerschbaum J., Bitter S., Weitlaner M., Kienzl-Wagner K., Neuwirt H., et al. Arterial Hypertension as a Risk Factor for Reduced Glomerular Filtration Rate after Living Kidney Donation. J Clin Med. 2020 Jan;9(2):338. doi:10.3390/jcm9020338

28. Lentine KL, Levey AS, Segev DL. Integrated Risk Assessment Versus Age-Specific GFR Thresholds for Living Donor Candidate Evaluation. Transplantation 2020 Mar. doi: 10.1097/TP.0000000000003243.

29. Keith DS, Brown J, Andreoni K. Living Donor Nephrectomy: Is It as Safe as It Can Be? Analysis of Living Donor Deaths in the United States. J Patient Saf 2019 Dec;15(4):274-281. doi: 10.1097/PTS.0000000000000610.

30. Burballa C, Crespo M, Redondo-Pachón D, Pérez-Sáez M.J, Mir M, Arias-Cabrales C et al. MDRD o CKDEPI en la estimación del filtrado glomerular del donante renal vivo. Nefrología 2018;38(2):207-212.

31. MacPhee A.M. I, DPhil, FRCP. Measured Versus Estimated Glomerular Filtration Rate in the Assessment of Living Kidney Donors: eGFR Has Its Limitations. Journal of Transplantation 2018 Oct;102(10):1595-1596.

32. Levey AS, Inker LA. GFR Evaluation in Living Kidney Donor Candidates. Journal of the American Society of Nephrology. 2017;28(4):1062-1071.

33. Fernández Fresnedo G, De la Oliva Valentín M. Guías S.E.N. Recomendaciones de la Sociedad Española de Nefrología (S.E.N.) y de la Organización Nacional de Trasplantes (ONT). Nefrología 2010;30(2):3-13.

34. Wahba R, Franke M, Hellmich M, Kleinert R, Cingöz T, Schmidt M.C, et al. Computed Tomography Volumetry in Preoperative Living Kidney Donor Assessment for Prediction of Split Renal Function. American Journal of Transplantation 2016;100(6):1270-1277.

35. Barbas A.S, Li Y, Zair M, Van J.A, Famure O, Dib M.J, Laurence J.M, et al. CT volumetry is superior to nuclear renography for prediction of residual kidney function in living donors. The Journal of Clinical and Translational Research 2016; 30(9):1028-1035.

36. Narváez Barros, A.; Riera Canals, L.; Alba Rey, E.; Alabat Roca, A.; Beato Garcia, S.; Fiol Riera, M.; Pujol Galarza, L.; Gil Vernet Cebrian, S.; Suarez Novo, J.F.; Vigués Julia, F. La volumetría renal mediante TC como método válido comparable al estudio isotópico (Tc99m-MAG3) para medir la función renal por separado en el estudio del donante vivo. XXVIII Reunión Nacional del Grupo de Trasplante. La Coruña, 2017.

37. Cherikh WS, Young CJ, Kramer BF, Taranto SE, Randall HB, Fan P-. Ethnic and Gender Related Differences in the Risk of End-Stage Renal Disease After Living Kidney Donation. American Journal of Transplantation 2011;11(8):1650-1655.

38. Organización Nacional De Trasplantes [sitio en Internet]. Memoria Actividad de Donación y Trasplante Renal España 2018. [Actualizado 5 Abril 2019] Disponible en: http://www.ont.es/infesp/Memorias/Actividad\%20de\%20Donaci\%C3\%B3n\%20y\%20Trasplante\%20Renal. pdf

39. Fehrman-Ekholm I, Kvarnström N, Söfteland J.M, Lennerling A, Rizell Magnus, Odén A et al. Postnephrectomy development of renal function in living kidney donors: a cross-sectional retrospective study. Nephrology Dialysis Transplantation. 2011 Jul; 26(7):2377-2381. https://doi.org/10.1093/ndt/gfr161 
40. Fenton A, Montgomery E, Nightingale P, Peters A.M, Sheerin N, Wroe A.C, et al. Glomerular filtration rate: new age- and gender- specific reference ranges and thresholds for living kidney donation. BMC Nephrology. 2018 Nov; 19:336.

41. Dols L.F.C, Kok N.F.M, Roodnat J.I, Tran T.C.K, Terkivatan T, Zuidema W.C, et al. Living Kidney Donors: Impact of Age on Long-Term Safety. American Journal of Transplantation. 2011 Apr; 11(4):737-742.

42. Poggio E.D, Rule A.D, Tanchanco R, Arrigain S, Butler R.S, Srinivas T, et al. Demographic and clinical characteristics associated with glomerular filtration rates in living kidney donors. Kidney International. 2009; 75(10):1079-1087.

43. Gaillard F, Legendre C, White CA. GFR Assessment of Living Kidney Donors Candidates.Transplantation. 2019 Feb 18. doi:10.1097/TP.0000000000002620.

44. Aggarwal N1, Porter AC, Tang IY, Becker BN, Akkina SK. Creatinine-based estimations of kidney function are unreliable in obese kidney donors. Transplant. 2012 Jan;2012:872894.

45. Mjøen G, Øyen O, Midtvedt K, Dahle DO, Norby G, Holdaas H. Age, gender, and body mass index are associated with renal function after kidney donation. Clin Transplant 2011; 25:579-583.

46. Unger L.W, Feka J, Sabler P, Rasoul-Rockenschaub S,Györi G, Hofmann M, et al. High BMI and male sex as risk factor for increased short-term renal impairment in living kidney donors - Retrospective analysis of 289 consecutive cases. International Journal of Surgery. 2017 Oct; 46:172-177.

47. Reese PP, Feldman HI, Asch DA, Thomasson A., Shults J., Bloom RD. Resultados a corto plazo para donantes de riñón vivos obesos y sus receptores. Trasplante. 2009; 88: 662-671.

48. Kerkeni W, Rebai M.H., Bouzouita A, Chakroun M, Slama R.B, Abdallah T.B et al. The effect of body mass index at the time of donation on postoperative and remote consequences of nephrectomy in 189 livingrelated kidney donors. Arab J Urol. 2015 Sep;13(3):221-4. doi: 10.1016/j.aju.2015.06.002.

49. Locke J.E, Reed R.D, Massie A, MacLennan P.A, Sawinski D, Kumar V et al. Obesity increases the risk of end-stage renal disease among living kidney donors. Kidney Int. 2017 Mar;91(3):699-703.

50. Godinho I, Guerra J, Melo M.J, Neves M, Gonçalves J, Santana M.A. et al. Living-Donor Kidney Transplantation: Donor-Recipient Function Correlation. Transplantation Proceedings. 2018 Apr;50(3):719722.

51. Pek GXW, Ngoh CLY, Teo BW, Vathsala A, Goh BYS, Young CHR et al. Visceral Obesity in Asian living kidney donors significantly impact early renal function after donor nephrectomy. World J Urol. 2018 Nov.

52. Guerra, J., Melo, M. J., Santana, A., Gonçalves, J., Nascimento, C., \& da Costa, A. G. (2015). Living Kidney Donor Transplantation: An Alternative With Limitations. Transplantation Proceedings. 2015 May; 47(4), 898-902.

53. Hamid R, Khan M, Aziz T, Mubarak M, Hashmi A, Hussain Z, et al. Short- and Intermediate-Term Outcomes in Living-Related Renal Transplant Donors. Exp Clin Transplant. 2018 Dec;16(6):656-659.

54. Registro Español de Trasplante Renal con Donante Vivo. Memoria de resultados 2010-2017. Disponible en: http://www.ont.es/infesp/Registros/INFORME\%20REGISTRO\%20DONANTE\%20VIVO\%2020102015_definitivo_Web.pdf

(C) 2020 por los autores; Esta obra está sujeta a la licencia de Reconocimiento 4.0 Internacional de Creative Commons. Para ver una copia de esta licencia, visite http://creativecommons.org/licenses/by-nc-nd/4.0/. 